\title{
Observer Based Tuning Techniques and Integrated SAW Torque Transducers for Two-Inertia Servo-Drive Systems
}

\author{
T. M. O’Sullivan, C. M. Bingham and N. Schofield* \\ THE UNIVERSITY OF SHEFFIELD \\ Department of Electronic and Electrical \\ Engineering, \\ University of Sheffield, Mappin Street, \\ Sheffield, S1 3JD, UK. \\ Tel.: +44 (0)1142225195 \\ Fax: $+44(0) 1142225196$ \\ E-Mail: t.osullivan@sheffield.ac.uk \\ URL: www.sheffield.ac.uk
}

\section{Keywords}

Servo-drive, Regulation, Motion control, Mechatronics, Noise, Industrial application,

\begin{abstract}
A controller design and tuning methodology is proposed that facilitates the rejection of periodic loadside disturbances applied to a torsional mechanical system, whilst simultaneously compensating for the disturbance observer's inherent phase delay, thereby facilitating the used of lower bandwidth, practically realisable, disturbance observers. The merits of implementing both a full- and reduced order observer, is investigated, with the latter being implemented with a new low-cost, high-bandwidth torque sensing device based on surface acoustic wave technology.
\end{abstract}

\section{Introduction}

Rejection of periodic load disturbances by feed-forward is a classical control problem when the torsional system interconnecting the motor and load can be considered infinitely stiff, and/or the dynamic load perturbations can be measured or estimated to a sufficiently high bandwidth [1]. In the majority of cases, load torque cannot be directly measured, and therefore, controllers usually require an observer (termed disturbance observer) to provide a dynamic estimate of the unknown input. Unfortunately, since feed-forward compensation is applied directly into the servo-amplifier, its effectiveness is heavily reliant on the ability of the observer to provide a high bandwidth, delay free, load torque signal, since any phase delay will significantly impede the ability of the controller to compensate effectively. However, observer algorithms are fundamentally derivative in nature (i.e. they amplify high frequency sensor noise), and those generally intended for industrial motion control applications (where usually the sole feedback sensor is the quantised position signal from an encoder) are therefore required to exhibit relatively low bandwidths, usually $<100 \mathrm{~Hz}$ [2], in order to attenuate high frequency noise and compensate for additional measurement delays [3]. Moreover, in such applications, the dynamics imparted by relatively low bandwidth observers unduly influences the system dynamics, ultimately affecting the desired rejection performance $[2,3]$. Nevertheless, for the sake of simplifying the theoretical analysis, state observer dynamics are assumed to be of a sufficiently high bandwidth that they can be ignored [1,4]. Few algorithms exist that compensate for the limitations imparted by a relatively low bandwidth observer, particularly in high performance motion control applications.

Furthermore, until recently, difficulties in acquiring reliable, low-noise, low-cost, shaft torque transducers that are non-invasive to the mechanical drive system, have precluded the use of direct shaft torque feedback for disturbance rejection in all but a minority of specialised closed-loop servodrive systems. Often, commonly employed torque transducers viz. strain gauge, optical and inductive 
devices, are too mechanically compliant when incorporated in a drive system, thereby degrading stability margins and reducing closed-loop bandwidth. Moreover, the additional cost associated with their integration is prohibitive. Here then, an investigation into improved tuning methodologies for classical disturbance observers, is investigated, with appraisal of a new, low-cost, non-contact torque measurement device, based on surface acoustic wave (SAW) technology [5]. SAW devices are mechanically robust, exhibit high sensitivity and bandwidth, and are largely unaffected by electromagnetic noise. They can be directly integrated into an electrical machine assembly without significantly affecting the mechanical stiffness of the motor shaft. For the study, SAW devices and RF module are mounted inside a commercial off-the-shelf permanent magnet synchronous machine (PMSM), directly onto the motor shaft between the front bearing and the rotor magnets, as illustrated in Fig 1.

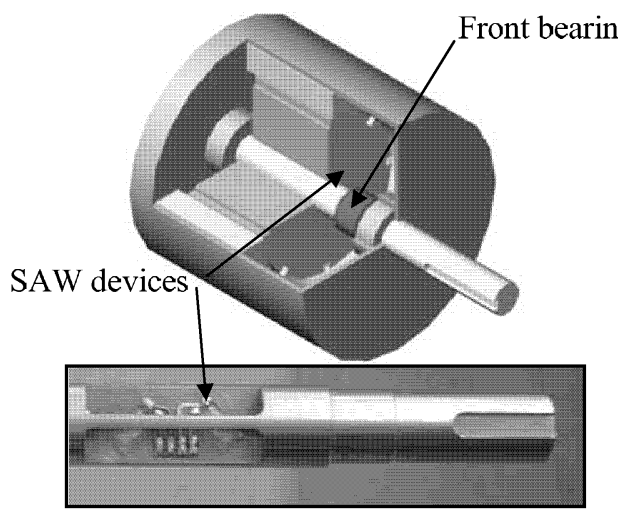

(a) illustration of servo-machine showing location of SAW devices and RF module

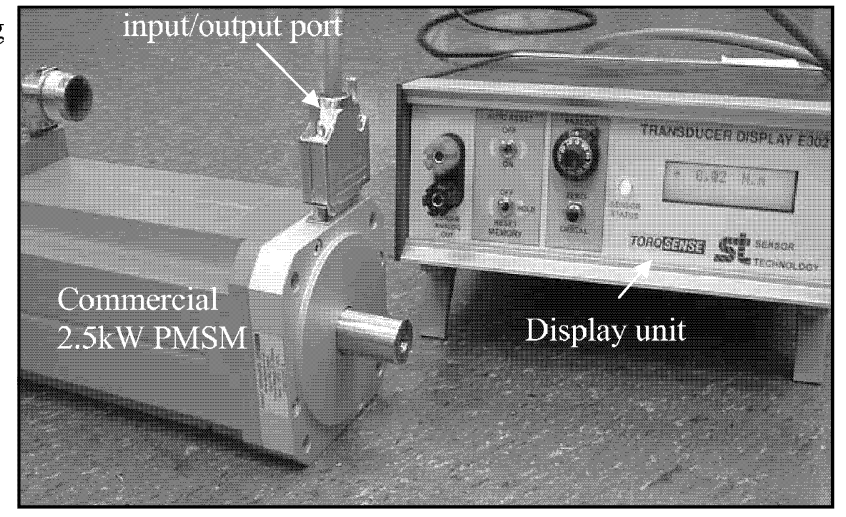

(b) Prototype servo-machine

Figure 1. PMSM with integrated 20Nm SAW torque transducer.

For simulation studies, a lumped two-inertia system model is used to represent the mechanical test facility that consists of $J_{m}$ and $J_{d}$ coupled via a shaft of finite stiffness $K_{m d}$, which is subject to torsional torque $t_{m d}$ and excited via the motor electromagnetic torque $t_{e}$, and load torque perturbations $t_{d}$. Similarly, the motor and load velocities are denoted by $\omega_{m}$ and $\omega_{d}$, respectively. Neglecting damping, $\omega_{a}$ is the anti-resonant frequency, $\omega_{n}$ the resonant frequency and $R$ the load-motor inertia ratio of the mechanical system, viz:

$$
\frac{\omega_{d}(s)}{t_{e}(s)}=\frac{\omega_{a}^{2}}{J_{m} s^{3}+J_{m} \omega_{n}{ }^{2} s} \quad \omega_{a}=\sqrt{K_{m d}\left(\frac{1}{J_{d}}\right)} \quad \omega_{n}=\omega_{a} \sqrt{R+1} \quad R=\frac{J_{d}}{J_{m}}
$$

A classical proportional and integral (PI) type control structure and two-inertia mechanical model is shown in Fig. 2(a). By augmenting this basic controller with feedback of additional state variables, enhanced disturbance rejection performance can be achieved. The general structure for such an extended controller is shown in Fig. 2(b), where $G(\mathrm{~s})$ represents the transfer function of the disturbance observer, $\hat{t}_{d}$ the observed disturbance torque and $K_{p d}, K_{d d}$, the associated transfer feedback gains. Furthermore, $K_{s}$ and $K_{d}$ represent the transfer feedback gains associated with shaft-torque and motor acceleration feedback, respectively. For example, setting $K_{p d}, K_{d d}$ and $K_{d}$ to zero will form a PI controller plus shaft torque feedback. This is commonly referred to as resonance ratio control (RRC) [6]. Similarly, setting $K_{p d}, K_{d d}$ and $K_{s}$ to zero will form a classical PI plus derivative (PID) controller [7] (i.e. implemented when a torque transducer is available). Moreover, both the RRC and PID controller structures can be additionally implemented with disturbance feedback, by setting the disturbance gains $K_{p d}, K_{d d}$ to a non-zero value. 


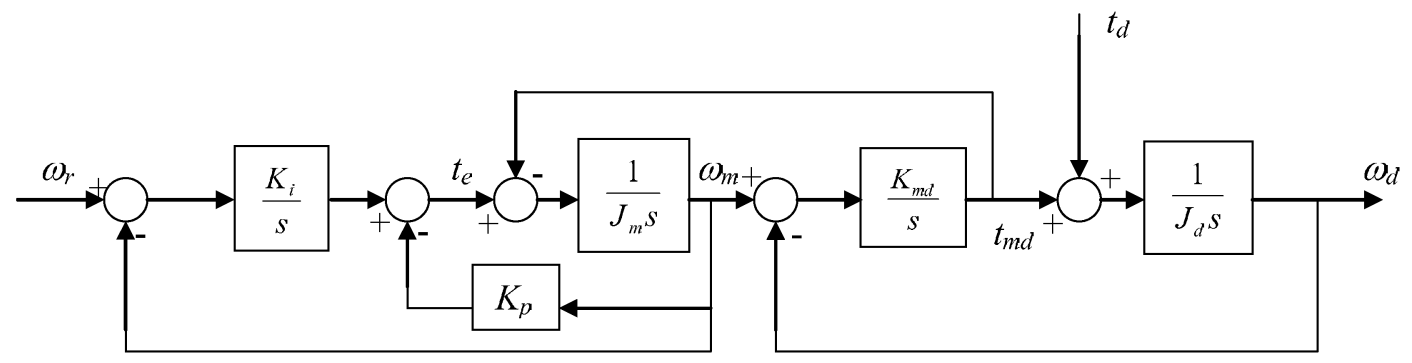

(a) Classical PI controller.

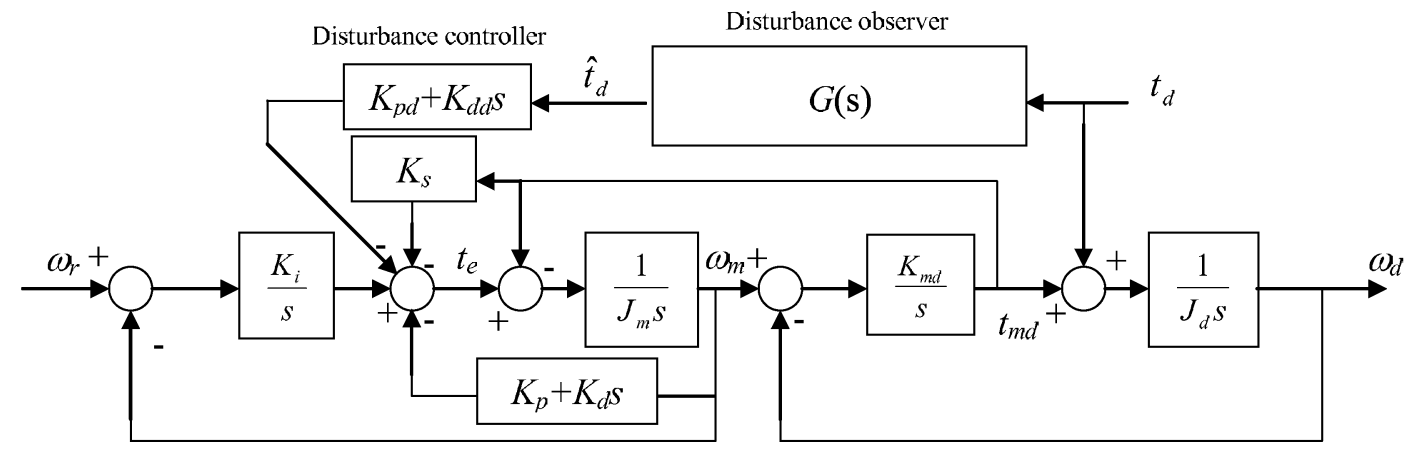

(b)Extended PI Controller augmented with feedback of additional state variables.

Figure 2. Controllers for two-inertia mechanical model.

\section{Disturbance Observer Structure}

Periodic load-side disturbances are a common feature of industrial automated production systems, for instance, where objects are dropped at equal time intervals onto a conveyer belt. For such systems, disturbance torque (as opposed to shaft or electromagnetic torque) cannot be sensed directly, and this requires an observer to provide a dynamic estimate. Assuming the disturbance torque is a statevariable, and is slow varying, implying that $\frac{d t_{d}}{d t} \rightarrow 0$, a state-variable representation of the two-inertia system can be obtained that includes the disturbance torque as a state, as follows:

$$
\frac{d}{d t}\left[\begin{array}{c}
\omega_{m} \\
\hdashline t_{m d} \\
\hdashline \omega_{d} \\
t_{d}
\end{array}\right]=\left[\begin{array}{c:c:cc}
0 & -\frac{1}{J_{m}} & 0 & 0 \\
\hdashline K_{m d} & 0 & K_{m d} & 0 \\
0 & \frac{1}{J_{d}} & 0 & -\frac{1}{J_{d}} \\
0 & 0 & 0 & 0
\end{array}\right] \cdot\left[\begin{array}{c}
\omega_{m} \\
\hdashline t_{m d} \\
\hdashline \omega_{d} \\
t_{d}
\end{array}\right]+\left[\begin{array}{c}
\frac{1}{J_{m}} \\
\hdashline 0 \\
\hdashline 0 \\
0
\end{array}\right] t_{e}
$$

Design of the state observer is based on Gopinath's method because of its ease of implementation [8]. To derive a suitable dynamic observer, the state vector is partitioned into two parts:

$$
\left[\begin{array}{c}
\dot{x}_{m} \\
\hdashline \dot{x}_{e}
\end{array}\right]=\left[\begin{array}{c:c}
A_{11} & A_{12} \\
\hdashline A_{21} & A_{22}
\end{array}\right] \cdot\left[\begin{array}{c}
x_{m} \\
\hdashline x_{e}
\end{array}\right]+\left[\begin{array}{c}
B_{1} \\
\hdashline B_{2}
\end{array}\right] u
$$


where, $x_{m}$ represents directly measured states, i.e. $x_{m}=y$, where $y$ is the measured outputs of the plant, and $x_{e}$ represents the remaining states that need to be observed. In the case of the PID controller, only the motor speed $\omega_{m}$ is sensed, and the required partitions are represented by dotted lines in (2). For the RRC controller, both $\omega_{m}$ and $t_{m d}$ are sensed, and the partitions are represented as dashed lines in (2). Notably, the order of the observer is reduced by the number of measured states. Hence, the PID controller requires a $3^{\text {rd }}$ order observer, and the RRC controller only requires a $2^{\text {nd }}$ order observer. For completeness, the dynamic structure of the observer is shown in Fig. 3, showing all the observed output states (only $\hat{t}_{d}$ is employed by the extended PID/RRC controllers).

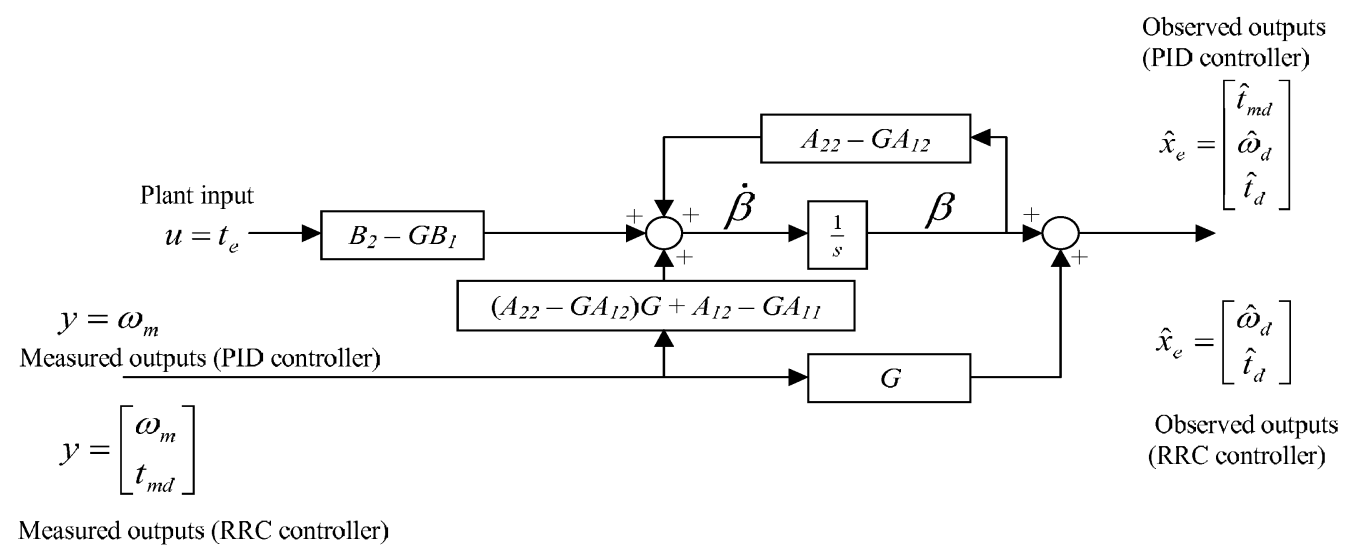

Figure 3. Block diagram representation of the observer structure.

The transfer function describing the relationship between the observed $\hat{t}_{d}$ and actual load-torque $t_{d}$ can be obtained from (3) [8], for the PID controller:

$$
G(s)^{P I D}=\frac{\hat{t}_{d}(s)}{t_{d}(s)}=\frac{-G_{3} \omega_{a}{ }^{2}}{J_{m} s^{3}-s^{2} G_{1}+s\left(\omega_{a}{ }^{2} J_{m}+G_{2} K_{m d}\right)-G_{3} \omega_{a}{ }^{2}}
$$

and the controller:

$$
G(s)^{R R C}=\frac{\hat{t}_{d}(s)}{t_{d}(s)}=\frac{G_{2} \omega_{a}{ }^{2}}{s^{2}-s G_{1} K_{m d}+G_{2} \omega_{a}^{2}}
$$

respectively, where the denominator equations describe the observer pole locations. The observer poles are therefore assigned according to the coefficients of the $2^{\text {nd }}$ and $3^{\text {rd }}$ optimal ITAE polynomials $[8,9]$ resulting in the optimal observer gains given in Table $\mathrm{I}$, where $\omega_{o b}$ is the equivalent $-3 \mathrm{~dB}$ observer bandwidth. If the observer bandwidth (and therefore the observer poles) is (are) assigned to be much greater than the highest frequency component of disturbance torque, it can be assumed that the observer does not unduly influence the assigned closed-loop regulation dynamics. Unfortunately, in practice, the observer structure is inherently derivative in nature and therefore sensitive to noisy input signals such as the motor speed signal that is obtained from the first derivative of quantised encoder position. As a result, therefore, the observer bandwidth must be minimised, since there exists a trade-off between the bandwidth of the observer and filtering of high frequency noise. Unfortunately, if consideration is not given to the additional dynamics imparted by a relatively low bandwidth disturbance observer, the closed-loop performance deteriorates as the observer bandwidth is reduced [9]. A tuning methodology is therefore proposed in the forgoing sections that facilitates the used of low bandwidth observes without sacrificing the control objectives. 
TABLE I. OPTIMAL OBSERVER AND CONTROLLER GAINS

\begin{tabular}{c|c|c|c}
\hline \multicolumn{2}{c|}{ DISTUBANCE OBSERVER } & \multicolumn{2}{c}{ CONTROLLER } \\
\hline $\begin{array}{c}\text { FULL-ORDER } \\
\text { (PID CONTROLLER) }\end{array}$ & $\begin{array}{c}\text { REDUCED-ORDER } \\
\text { (RRC CONTROLLER) }\end{array}$ & PID & RRC \\
\hline$G_{1}=-1.75 \omega_{o b} J_{m}$ & $G_{1}=\frac{-1.4 \omega_{o b}}{K_{m d}}$ & $K_{p} \approx 1.85 \omega_{a} J_{d}$ & $K_{p} \approx 1.85 \omega_{a} J_{m}$ \\
$G_{2}=\frac{\left(2.15 \omega_{o b}{ }^{2}-\omega_{a}{ }^{2}\right) J_{m}}{K_{m d}}$ & $G_{2}=\frac{\omega_{o b}{ }^{2}}{\omega_{a}{ }^{2}}$ & $K_{i} \approx 0.6 \omega_{a}{ }^{2} J_{d}$ & $K_{i} \approx 0.6 \omega_{a}{ }^{2} J_{m}$ \\
$G_{3}=\frac{-\omega_{o b}{ }^{3} J_{m}}{\omega_{a}{ }^{2}}$ & & $K_{d}=J_{d}-J_{m}$ & $K_{s}=\frac{J_{m}}{J_{d}}-1$ \\
\hline
\end{tabular}

\section{PID/RRC with Disturbance Feedback}

Equations (6) and (7) provide the closed-loop transfer functions describing the regulation dynamics $\left(\omega_{d}(\mathrm{~s}) / t_{d}(\mathrm{~s})\right)$ for the PID and RRC controllers with disturbance feedback, respectively. For the PID controller (6), it can be seen that the numerator is $5^{\text {th }}$ order (excluding the $\mathrm{s} / J_{d}$ multiplier term) which is comprised of a real zero and two pairs of complex zeros that can be assigned by the selection of the observer bandwidth, $\omega_{o b}$ and the disturbance feedback gains $K_{p d}$ and $K_{d d}$. Similarly, for the RRC controller (7), the numerator is $4^{\text {th }}$ order which is comprised of two pairs of complex zeros that can be assigned. Thus, in both cases, by proper adjustment of these gains, a pair of complex conjugate zeros are assigned to the imaginary axis (no damping) for a user-defined frequency, $\omega_{r j}$, thereby rejecting periodic load-side disturbances of that frequency, whilst eliminating the effects of the observer dynamics on the rejection performance, i.e. a relatively low bandwidth disturbance observer can be implemented, attenuating high frequency noise, without sacrificing the control objective. However, to impart adequate closed-loop damping whilst additionally rejecting periodic load disturbances (a common industrial requirement), it is desirable to have the ability to independently assign both the closed-loop poles and zeros simultaneously. The closed-loop poles, when disturbance feedback is not implemented (denoted the first bracket $4^{\text {th }}$ order polynomial expression in the denominator of $(6)$ and (7)) are independently assigned according to the coefficients of the optimal $4^{\text {th }}$ order ITAE polynomial [8], by the controller gains in Table I [9,10]. Moreover, it has been shown that the basic PI controller and two-inertia model imparts optimum closed-loop damping performance when $R \approx 1$ (according to the optimal ITAE polynomial) and increasingly under damped responses for $R<1$ [7,9,10]. Moreover, the extra degree of freedom afforded by the PID and RRC controller enables $R$ to be virtually adjusted to $\tilde{R} \approx 1$ by adjusting $K_{d}$ or $K_{s}$, respectively- see Table I.

$$
\begin{aligned}
& \frac{\omega_{d}}{t_{d}}=
\end{aligned}
$$

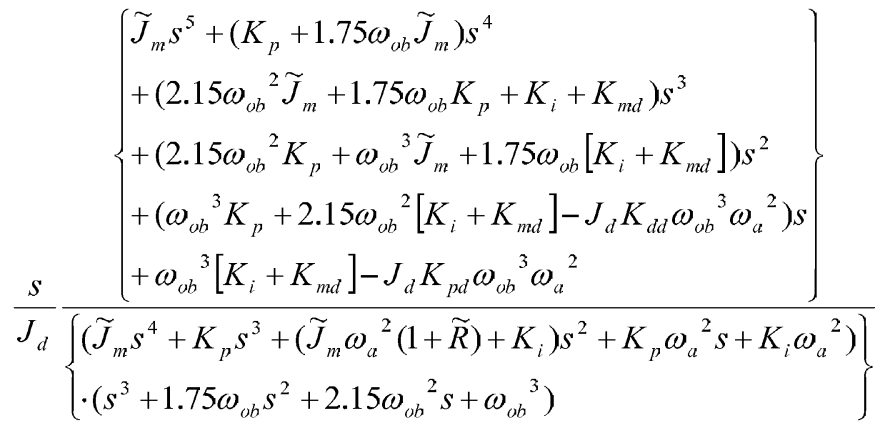

$$
\text { where } \tilde{J}_{m}=J_{m}+K_{d} \text { and } \tilde{R}=J_{d} / \tilde{J}_{m}
$$




$$
\begin{aligned}
& \frac{\omega_{d d}}{t_{d}}= \\
& \frac{s}{J_{d}} \frac{\left\{\begin{array}{l}
J_{m} s^{4}+\left(K_{p}+1.4 \omega_{o b} J_{m}\right) s^{3} \\
+\left(\omega_{o b}{ }^{2} J_{m}+1.4 \omega_{o b} K_{p}+\left[K_{i}+K_{m d}\left(1+K_{s}\right)\right]\right) s^{2} \\
+\left(\omega_{o b}{ }^{2} K_{p}+1.4 \omega_{o b}\left[K_{i}+K_{m d}\left(1+K_{s}\right)\right]-J_{d} K_{d d} \omega_{o b}{ }^{2} \omega_{a b}{ }^{2}\right) s \\
+\omega_{o b}{ }^{2}\left[K_{i}+K_{m d}\left(1+K_{s}\right)\right]-J_{d} K_{p d} \omega_{o b}{ }^{2} \omega_{a}{ }^{2}
\end{array}\right\}}{\left\{\begin{array}{l}
\left(J_{m} s^{4}+K_{p} s^{3}+\left(J_{m} \omega_{a}{ }^{2}(1+\widetilde{R})+K_{i}\right) s^{2}+K_{p} \omega_{a}{ }^{2} s+K_{i} \omega_{a}{ }^{2}\right) \\
\cdot\left(s^{2}+1.4 \omega_{o b} s+\omega_{o b}{ }^{2}\right)
\end{array}\right\}} \\
& \text { where } \tilde{R}=R\left(1+K_{s}\right)
\end{aligned}
$$

Equation (8) shows a $5^{\text {th }}$ order polynomial expression factored into two parts. The first, a complex root, represents the user defined rejection frequency, $\omega_{r j}$, where the damping ratio equals zero. The second factor, a $3^{\text {rd }}$ order polynomial expression, defines the arbitrary location of the other roots.

$$
\begin{aligned}
& \left(s^{2}+{\omega_{r j}}^{2}\right)\left(s^{3}+a s^{2}+b s+c\right) \\
& =s^{5}+s^{4} a+s^{3}\left(b+\omega_{r j}\right)+s^{2}\left(c+{\omega_{r j}}^{2} a\right)+s b{\omega_{r j}}^{2}+c{\omega_{r j}}^{2}
\end{aligned}
$$

By equating the numerator of (6) with the expanded polynomial in (8), expressions for the disturbance feedback gains $K_{p d}$ and $K_{d d}$ can be derived for the PID controller that enables $\omega_{r j}$ and the observer bandwidth to be independently assigned, as follows:

$$
\begin{aligned}
& K_{p d}=\frac{\omega_{o b}{ }^{3}\left(K_{i}+K_{m d}\right)-\omega_{r j}{ }^{2}\left(\widetilde{J}_{m} \omega_{o b}{ }^{3}+2.15 \omega_{o b}{ }^{2} K_{p}+1.75 \omega_{o b}\left(K_{i}+K_{m d}\right)-\omega_{r j}{ }^{2}\left(\widetilde{J}_{m} \omega_{o b} 1.75+K_{p}\right)\right)}{J_{d} \omega_{o b}{ }^{3} \omega_{a}{ }^{2}} \\
& K_{d d}=\frac{\omega_{o b}{ }^{3} K_{p}+2.15 \omega_{o b}{ }^{2}\left(K_{i}+K_{m d}\right)-\omega_{r j}{ }^{2}\left(\widetilde{J}_{m} 2.15 \omega_{o b}{ }^{2}+1.75 \omega_{o b} K_{p}+K_{i}+K_{m d}-\omega_{r j}{ }^{2} \widetilde{J}_{m}\right)}{J_{d} \omega_{o b}{ }^{3} \omega_{a}{ }^{2}}
\end{aligned}
$$

Similarly, for the controller, the numerator of (7) is equated to a factorised $4^{\text {th }}$ order polynomial expression:

$$
\begin{aligned}
& \left(s^{2}+{\omega_{r j}}^{2}\right)\left(s^{2}+b s+c\right) \\
& =s^{4}+s^{3} b+s^{2}\left(c+\omega_{r j}{ }^{2}\right)+s{\omega_{r j}}^{2} b+c \omega_{r j}{ }^{2}
\end{aligned}
$$

giving the following disturbance feedback gains:

$$
K_{p l}=\frac{\omega_{o b}{ }^{2}\left(K_{i}+K_{m d}\left(1+K_{s}\right)\right)-\omega_{r j}{ }^{2}\left(\omega_{o b}{ }^{2} J_{m}+1.4 \omega_{o b} K_{p}+K_{i}+K_{m d}\left(1+K_{s}\right)-\omega_{r j}{ }^{2} J_{m}\right)}{J_{d} \omega_{o b}{ }^{2} \omega_{a l}{ }^{2}}
$$




$$
K_{d d}=\frac{\omega_{o b}{ }^{2} K_{p}+1.4 \omega_{o b}\left(K_{i}+K_{m d}\left(1+K_{s}\right)\right)-\omega_{r j}{ }^{2}\left(K_{p}+1.4 \omega_{o b} J_{m}\right)}{J_{d} \omega_{o b}{ }^{2} \omega_{a}{ }^{2}}
$$

By way of example, the user defined rejection frequency is set at $\omega_{r j}=62.8 \mathrm{rad}(10 \mathrm{~Hz})$ with the mechanical parameters defined in Table II. Figure 4 shows the regulation bode magnitude plots for the PID and RRC controller, respectively, as $\omega_{o b}$ is adjusted. It can be seen that, for both controllers, reducing the observer bandwidth does not significantly influence the control objective, i.e. the attenuation at $\omega_{r j}$ remains constant.

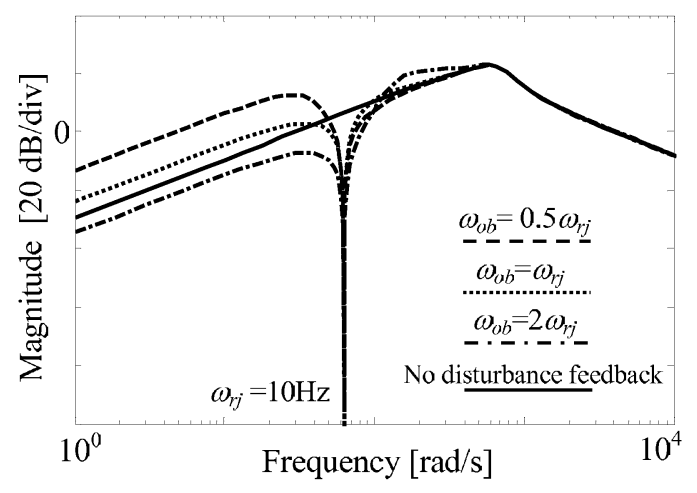

(a) PID controller

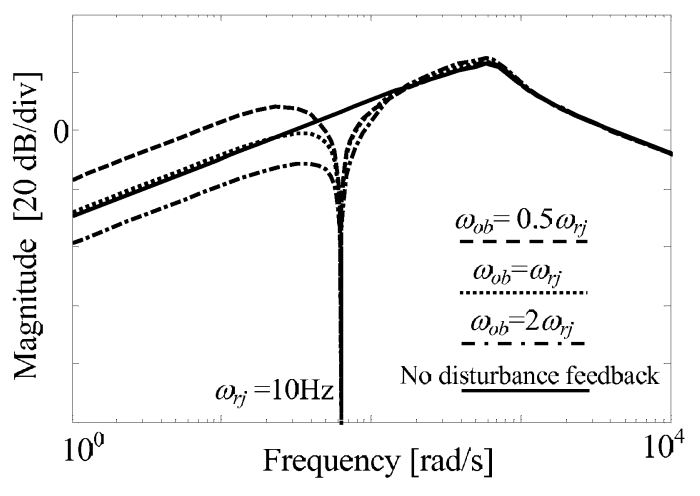

(b) RRC controller

Figure 4. Regulation performance of proposed controller structures and tuning methodology

However, it can be seen that, in general, the RRC controller improves low frequency attenuation for a given observer bandwidth, when compared to that of the PID controller. It should be noted that for both controllers, the observer bandwidth cannot be made infinitely small, since, for low bandwidth observers, low frequency disturbances are amplified when compared to case with no disturbance feedback (i.e. when $K_{p d}, K_{d d}=0$ ), as evidenced in Fig 4. It is therefore desirable to employ an observer of sufficient relative bandwidth to ensure adequate rejection of the low frequency band, particularly in the event of a variation in the disturbance frequency.

\section{Experimental Validation}

The proposed control techniques, simulation results and observations are now validated on the experimental test-facility, comprising of the $2.2 \mathrm{~kW}$ prototype PMSM with integrated $20 \mathrm{Nm}$ SAWbased torque transducer, Fig. 1(b), and a similarly rated loading machine (representing the motor and load inertias, $J_{m}$ and $J_{d}$, respectively). The machines are coupled back-to-back via a shaft and couplings. Once again, Table II gives the resulting mechanical parameters.

The experimental results now presented are in response to a $3 \mathrm{Nm}$ sinusoidal disturbance torque with a frequency of $62.8 \mathrm{rad} / \mathrm{s}(10 \mathrm{~Hz})$, where the speed controller reference is chosen to be constant at $10 \mathrm{rad} / \mathrm{s}$. For all results, shaft torque measurements are obtained from the integrated torque transducer. Figure 5 shows the time domain regulation performance imparted by the PID controller with no disturbance feedback. It can be seen that the oscillatory load torque induces a corresponding unwanted oscillatory component of load-side speed. 

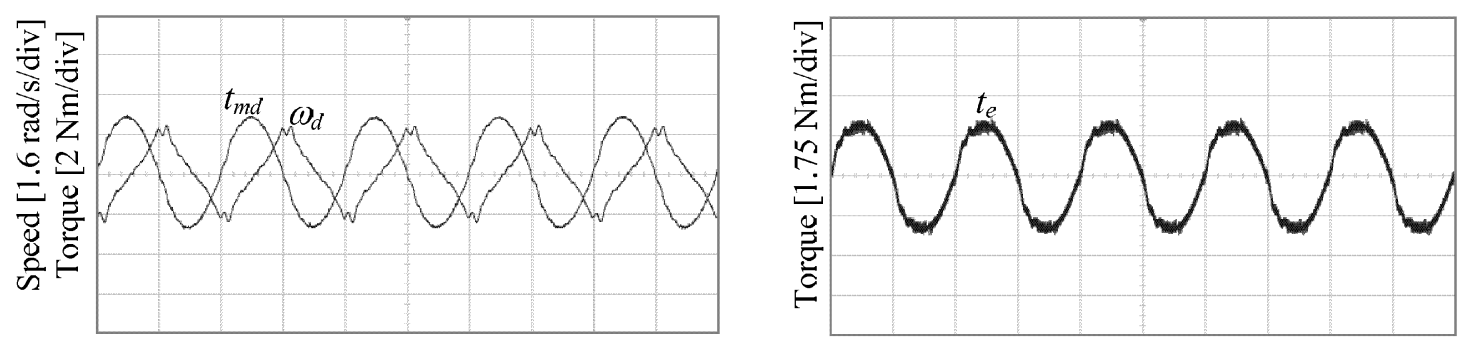

Time $[50 \mathrm{~ms} / \mathrm{div}]$

Figure 5. PID controller with without disturbance torque feedback.

Figure 6 now shows the PID controller responses with disturbance feedback, as the observer bandwidth is increased, where $\omega_{o b}=0.5 \omega_{r j}, \omega_{r j}$ and $1.25 \omega_{r j}$, respectively. It can be seen that the load perturbations are rejected from the load-speed, and rejection performance is not unduly influenced by the bandwidth of the observer. Furthermore, when the observer bandwidth is less than the rejection frequency, Fig 5.10(a), i.e. when $\omega_{o b}=0.5 \omega_{r j}$, the controller noise is attenuated to levels comparable to that obtained with no disturbance feedback, Fig. 6 .

\begin{tabular}{l|l}
\multicolumn{2}{c}{ TABLE II } \\
\multicolumn{2}{c}{ MECHANICAL PARAMETERS } \\
\hline$R$ & 0.5 \\
$J_{d}$ & $0.00025 \mathrm{kgm}^{2}$ \\
$K_{m d}$ & $80 \mathrm{Nm} / \mathrm{rad}$ \\
$\omega_{a}$ & $565.7 \mathrm{rad} / \mathrm{s}(90 \mathrm{~Hz})$ \\
\hline
\end{tabular}

Notably, when $\omega_{o b}>1.5 \omega_{r j}(>15 \mathrm{~Hz})$ instability occurred when using the PID controller due to the elevated noise levels. This is a result of the requirement of a higher order disturbance observer (compared with the RRC controller), thereby exacerbating the effects of high frequency noise originating from the motor speed signal. Furthermore, the PID controller requires a motor acceleration signal estimated via the double-derivative of the encoder position. By comparison, the RRC controller can accommodate a significantly higher bandwidth observer before instability occurs (thereby ensuring adequate rejection of all low frequencies in the event of the rejection frequency varyingsee Fig. 4), when $\omega_{o b}>3.5 \omega_{r j}(>35 \mathrm{~Hz})$, by virtue of the reduced noise levels. By way of example, Fig. 7 shows the responses of the RRC controller for $\omega_{o b}=3 \omega_{r j}$, and $2 \omega_{r j}$, respectively

Furthermore, Figs. 6 and 7 demonstrate the equivalence of the PID and RRC controllers with observer disturbance feedback. However, by virtue of increased observer bandwidth, and improved rejection performance, (compare Figs 4(a) and (b)) the RRC controller, in general, imparts improved disturbance rejection over the low frequency band resulting in 'flatter' unperturbed load speed traces, as evidenced in Fig 7.

\section{Conclusion}

To address the effects of periodic load-side disturbance torques on the load speed profile, extended controllers, based on classical feed-forward compensation, have been proposed. For the investigation, a SAW torque transducer is mounted inside a commercial off-the-shelf permanent magnet synchronous machine. Specifically, both PID and RRC controllers are augmented with an additional feedback of observed disturbance torque (since it cannot be measured), via a proportional and derivative based controller. The extended controllers afford the flexibility to simultaneously impart optimal closed-loop damping, by allowing the independent selection of virtual inertia ratio, whilst 
additionally, facilitating the independent assignment of regulation transmission zero's, such that, when properly assigned, enable the rejection of a periodic disturbance torque from the load speed.
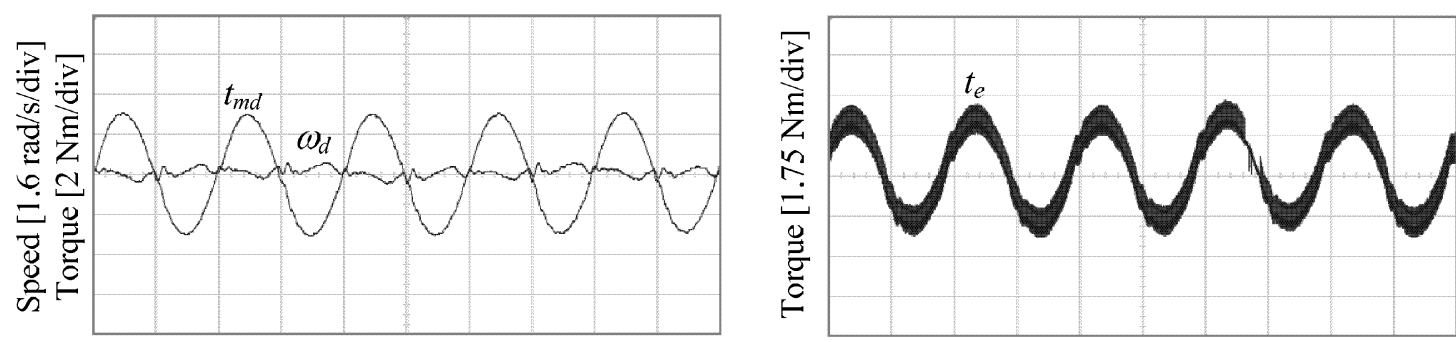

Time $[50 \mathrm{~ms} / \mathrm{div}]$

(a) $\omega_{o b}=0.5 \omega_{r j}$
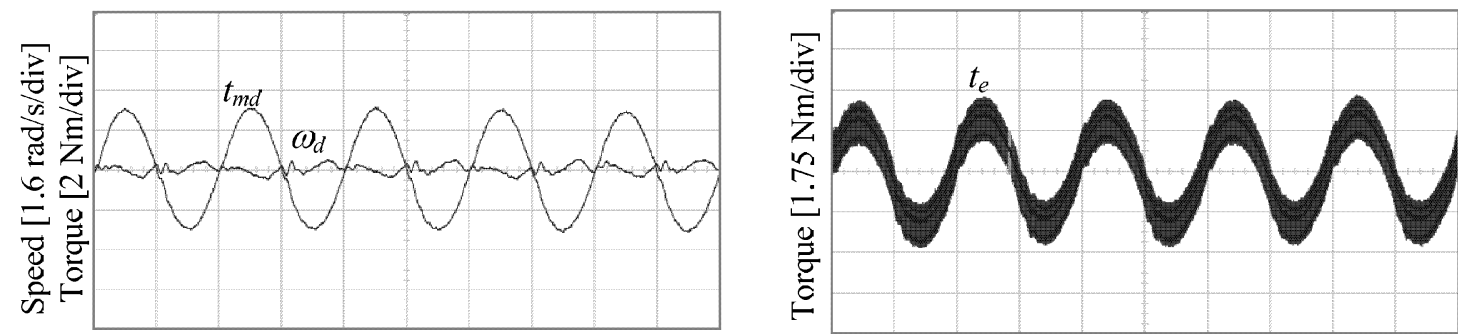

Time $[50 \mathrm{~ms} / \mathrm{div}]$

(b) $\omega_{o b}=\omega_{r j}$
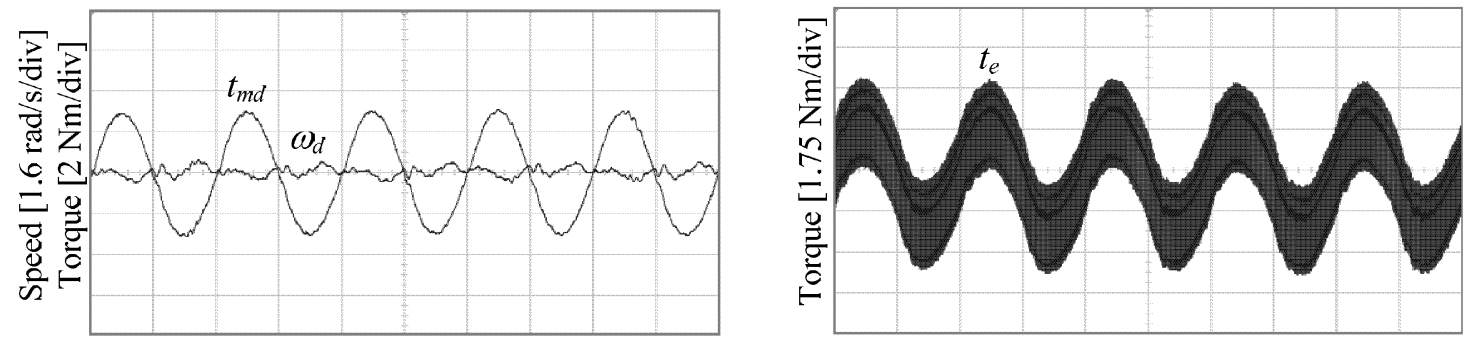

Time $[50 \mathrm{~ms} / \mathrm{div}]$

(c) $\omega_{o b}=1.25 \omega_{r j}$

Figure 6. PID controller with observed disturbance torque feedback.

Moreover, to realise disturbance feedback, the PID controller requires a full, third-order disturbance observer, since only motor position feedback is employed, whereas the RRC controller, which employs both shaft torque and motor position sensors, can be implemented with a second-order observer. Appraisal has been given to the combined observer and controller dynamics, with the zeros reassigned, demonstrating, for the first time, that the bandwidth of the observer need not effect the rejection of a periodic disturbance, thereby enabling significantly lower bandwidth observers to be employed, i.e. the observer bandwidth can be lower than the rejection frequency. It is shown that higher bandwidth disturbance observers are required to maximise the disturbance attenuation over all of the low frequency band (as well as the desired rejection frequency), thereby attenuating a wide range of possible frequencies. In such cases, therefore, it is shown that the RRC controller is the 
preferred solution by virtue of reduced noise sensitivity. Furthermore, it is demonstrated that a integrated $20 \mathrm{Nm}$ SAW torque transducer, as employed by the RRC controller, is not unduly affected by machine generated electromagnetic noise. Additionally, since the SAW devices are inherently low cost in mass production, and the mechanical modifications to the commercial PMSM, are minimal, the cost of high volume integration is estimated to increase the total machine cost by only a few percent.
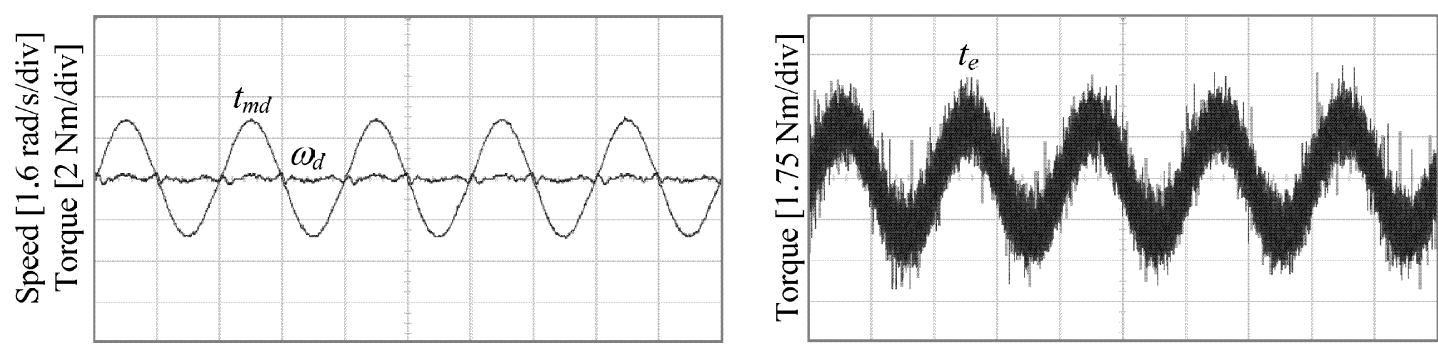

Time $[50 \mathrm{~ms} / \mathrm{div}]$

(a) $\omega_{\mathrm{ob}}=3 \omega_{\mathrm{rj}}$
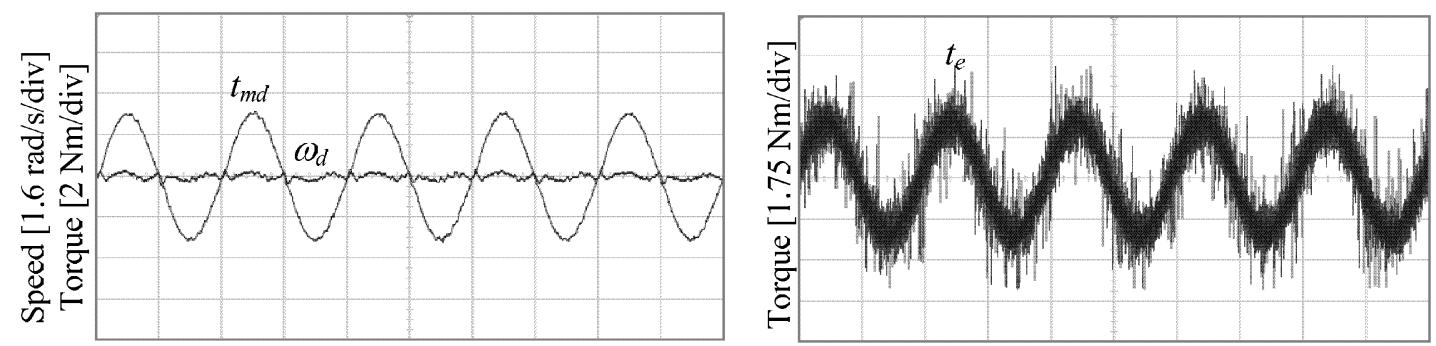

Time $[50 \mathrm{~ms} / \mathrm{div}]$

(b) $\omega_{o b}=2 \omega_{r j}$

Figure 7. RRC controller with observed disturbance torque feedback.

\section{References}

[1]G. Ellis, "Observers in control systems: A practical guide," Book, Academic Press, ISBN: 0-12-237472-X, 2002.

[2]S. N. Vukosavic and M. R. Stojic, "Suppression of torsional oscillations in a high performance speed servo drive," IEEE Transactions on Industrial Electronics, Vol. 45, No. 1, pp. 108-117, February 1998.

[3]K. Hong, "A load torque compensation scheme under the speed measurement delay," IEEE transactions on industrial electronics, Vol. 45, No. 2, April 1998.

[4]J.K. Ji and S. K. Sul, "Kalman filter and LQ based speed controller for torsional vibration suppression in a 2-mass motor drive system," IEEE Transactions on Industrial Electronics, Vol. 42, No. 6, pp. 564-571, December 1995.

[5] A. Lonsdale, "Dynamic rotary torque measurement using surface acoustic waves," Sensors, Vol. 18, no. 10, Oct. 2001, pp. 51-56.

[6]Y. Hori, H. Sawada and Y. Chun, "Slow resonance ratio control for vibration suppression and disturbance rejection in torsional system," IEEE Transactions on Industrial Electronics, Vol. 46, No. 1, pp. 162-8, February 1999.

[7]G. Zhang, J. Furusho "Speed control of a two-inertia system by PI/PID control," IEEE Transactions on Industrial Electronics, Vol. 47, No. 3, pp. 603-609, June 2000.

[8]G. Franklin, J. D. Powell and A. Emami-Naeini "Feedback Control of Dynamic Systems", Book, Prentice-Hall, ISBN: 013-032393-4, 2002.

[9]T. O'Sullivan, N. Schofield, C. M. Bingham, "SAW torque transducers for disturbance rejection and tracking control of multi-inertia servo-drive systems," Power Electronics Specialists Conference (PESC04), Vol. 6, pp. 4578-4584, 2004.

[10]T. O’Sullivan, N. Schofield and C.M. Bingham, "High-performance control of dual-inertia servo-drive systems using low-cost integrated SAW torque transducers," Accepted for publication, IEEE Industrial Electronics, 2005. 